\title{
A Note on the Unsteady Incompressible MHD Fluid Flow with Slip Conditions and Porous Walls
}

\author{
H. Zaman, ${ }^{1}$ Z. Ahmad, ${ }^{1}$ and M. Ayub ${ }^{2}$ \\ ${ }^{1}$ Department of Mathematics, Islamia College Chartered University, Peshawar 25000, Pakistan \\ ${ }^{2}$ Department of Mathematics, Quaid-i-Azam University, Islamabad 45320, Pakistan \\ Correspondence should be addressed to H. Zaman; haiderzaman67@yahoo.com
}

Received 16 July 2013; Accepted 3 October 2013

Academic Editors: A. M. Gavrilik and A. Herrera-Aguilar

Copyright (c) $2013 \mathrm{H}$. Zaman et al. This is an open access article distributed under the Creative Commons Attribution License, which permits unrestricted use, distribution, and reproduction in any medium, provided the original work is properly cited.

\begin{abstract}
This work is concerned with the influence of uniform suction or injection on flow and heat transfer analysis of unsteady incompressible magnetohydrodynamic (MHD) fluid with slip conditions. The resulting unsteady problem for velocity and heat transfer is solved by means of Laplace transform. The characteristics of the transient velocity, overall transient velocity, steady state velocity and heat transfer at the walls are analyzed and discussed. Graphical results reveal that the magnetic field, slip parameter, and suction (injection) have significant influences on the velocity, and temperature distributions, which also changes the heat transfer behaviors at the two plates. The results of Fang (2004) are also recovered by keeping magnetic field and slip parameter absent.
\end{abstract}

\section{Introduction}

Navier-Stokes equations are the basic equations of fluid mechanics. Exact solutions of Navier-Stokes equations are rare due to their inherent nonlinearity. Exact solutions are important because they serve as accuracy checks for numerical solutions. Complete integration of these equations is done by computer techniques, but the accuracy of the results can be established only by comparison with exact solutions. In the literature, there are a large number of Newtonian fluid flows for which exact solutions are possible [1-6]. The effects of transverse magnetic field on the flow of an electrically conducting viscous fluid have been studied extensively in view of numerous applications to astrophysical, geophysical, and engineering problems [7-15]. If the working fluid contains concentrated suspensions, then the wall slip can occur [16]. Khaled and Vafai [3] studied the effect of the slip on Stokes and Couette flows due to an oscillating wall. However, the literature lacks studies that take into account the possibility of fluid slippage at the walls. Applications of these problems appear in microchannels or nanochannels and in applications where a thin film of light oil is attached to the moving plates or when the surface is coated with special coating such as a thick monolayer of hydrophobic octadecyltrichlorosilane [17]. Yu and Ameel [18] imposed nonlinear slip boundary conditions on flow in rectangular microchannels. Erdogan [6] studied deeply the solution to the Stokes problem under nonslip conditions at the wall. Ayub and Zaman [19] studied the effects of suction and blowing for orthogonal flow impinging on a wall. Khan et al. [20] discussed the flow of Sisko fluid through a porous medium. Ariel et al. [21] considered the flow of elasticoviscous fluid with partial slip. Raptis et al. [22-25] studied steady and unsteady free convection and mass transfer flow through a porous medium. Penton [26] presented the transient solution for the flow due to the oscillating plate.

In this note, the flow of an incompressible, unsteady, viscous, MHD fluid with slip conditions is considered. Unsteady means time dependent flow, and we are looking for the effects of different parameters on flow with the variation of time. Unsteady and steady velocity profiles with mass transfer will be presented and solved exactly. There is mass injection from one plate and the same amount of suction on the other plate. The steady state temperature is also solved and discussed. When the fluid motion is set up from rest, the velocity field contains transients, determined by the initial conditions which gradually disappear in time. The effect of magnetic field and time on the transient 
velocity and on overall transient velocity has been seen graphically for both injection and suction. The effect of slip parameter on steady state velocity for injection/suction is shown graphically. Steady state temperature profiles and heat transfer rate at the walls (Nusselt number at the walls) are also discussed for injection/suction for different Prandtl and Reynolds numbers. The results of Fang [1] are recovered by taking magnetic field parameter and slip parameter to be zero.

\section{Theoretical Derivation}

2.1. Transient Velocity Profiles. Consider an incompressible, viscous, unsteady flow problem, in which there is slip between the bottom wall and fluid and also between top wall and fluid. There are mass injection velocity $v_{w}$ at the bottom wall and mass suction velocity $v_{w}$ at the top wall; $v_{w}>$ 0 corresponds to injection and $v_{w}<0$ corresponds to suction. The governing equation for this problem can be obtained as [2]

$$
\frac{\partial u(y, t)}{\partial t}+v_{w} \frac{\partial u(y, t)}{\partial y}=v \frac{\partial^{2} u(y, t)}{\partial y^{2}}-N u(y, t),
$$

where $u(y, t)$ is the velocity of the fluid in the $y$-direction which is along the wall direction, $y$ is the distance alon $y$ axis $g, t$ is the time, $v=\mu / \rho, N=\sigma B_{0}^{2} / \rho, \mu$ is the dynamic viscosity, $\nu$ is the kinematic viscosity, $\rho$ is the density of the fluid, $\sigma$ is the electric conductivity of the fluid, $B_{0}$ is the applied magnetic field, and $N$ is the MHD factor or parameter. For the boundary conditions we consider the existence of slip between the velocity of the fluid at the walls and speed of the walls:

$$
\begin{gathered}
u(0, t)-\left.\lambda \frac{\partial u(y, t)}{\partial y}\right|_{y=0}=0, \\
u(h, t)+\left.\lambda \frac{\partial u(y, t)}{\partial y}\right|_{y=h}=U_{0} .
\end{gathered}
$$

Initial condition is

$$
u(y, 0)=0,
$$

where $\lambda$ is the slip parameter $(\lambda=0$ gives the usual no slip condition at the wall) and $U_{0}$ is the velocity at the upper wall. The problem exactly reduces to the problem of Fang [1] if we take $N=0, \lambda=0$. Equations (1), (2), and (3) can be made dimensionless by defining

$$
U=\frac{u}{U_{0}}, \quad Y=\frac{y}{h}, \quad T=\frac{t}{\tau_{c}}=\frac{t \nu}{h^{2}} .
$$

Then (1), (2), and (3) become

$$
\begin{gathered}
\frac{\partial U}{\partial T}+R_{e} \frac{\partial U}{\partial Y}=\frac{\partial^{2} U}{\partial Y^{2}}-\bar{N} U, \\
U(0, T)-\left.\bar{\lambda} \frac{\partial U(Y, T)}{\partial Y}\right|_{Y=0}=0, \\
U(1, T)+\left.\bar{\lambda} \frac{\partial U(Y, T)}{\partial Y}\right|_{Y=1}=1, \\
U(Y, 0)=0,
\end{gathered}
$$

where $\bar{N}=N\left(h^{2} / \nu\right), \bar{\lambda}=\lambda / h$, and $R_{e}=v_{w}(h / \nu)$ (Reynolds number). Decomposing $U(Y, T)$ into two parts, say, transient part and a steady state part,

$$
U(Y, T)=U_{t}(Y, T)+U_{s}(Y, T) .
$$

Then we have two separate problems and the steady state part will be

$$
\begin{gathered}
R_{e} \frac{d U_{s}}{d Y}=\frac{d^{2} U_{s}}{d Y^{2}}-\bar{N} U_{s}, \\
U_{s}(0, T)-\left.\bar{\lambda} \frac{\partial U_{s}(Y, T)}{\partial Y}\right|_{Y=0}=0 \\
U_{s}(1, T)+\left.\bar{\lambda} \frac{\partial U_{s}(Y, T)}{\partial Y}\right|_{Y=1}=1 .
\end{gathered}
$$

The solution of (10) with BCs (11) and (12) can be obtained as

$$
U_{s}(Y)=\frac{e^{a Y}\left[(1-\bar{\lambda} a) \sinh \left(b_{1} Y\right)+\bar{\lambda} b_{1} \cosh \left(b_{1} Y\right)\right]}{e^{a}\left[\left(1-\bar{\lambda}^{2}\left(a^{2}-b_{1}^{2}\right)\right) \sinh \left(b_{1}\right)+2 \bar{\lambda} b_{1} \cosh \left(b_{1}\right)\right]},
$$

where $a=R_{e} / 2$ and $b_{1}=\sqrt{R_{e}^{2} / 4+\bar{N}}$. When $\bar{\lambda}=0$ (no slip) and $\bar{N}=0$ (no magnetic field), then (13) becomes

$$
U_{s}(Y)=\frac{e^{\left(R_{e} / 2\right) Y} \sinh \left(R_{e} / 2\right) Y}{e^{R_{e} / 2} \sinh \left(R_{e} / 2\right)}=\frac{1-e^{R_{e} Y}}{1-e^{R_{e}}} .
$$

Equation (14) the result number (7) of Fang [1]. If there is no mass transfer at the walls, then $R_{e}=0$, so $a=0$ and $b_{1}=$ $\sqrt{\bar{N}}$, and (13) becomes

$$
U_{s}(Y)=\frac{\sinh (\sqrt{\bar{N}} Y)+\bar{\lambda} \sqrt{\bar{N}} \cosh (\sqrt{\bar{N}} Y)}{\left(1+\bar{\lambda}^{2} \bar{N}\right) \sinh (\sqrt{\bar{N}})+2 \bar{\lambda} \sqrt{\bar{N}} \cosh (\sqrt{\bar{N}})} .
$$

If there is no mass transfer at the walls and magnetic field is absent, then $R_{e}=0, \bar{N}=0$, and slip parameter $\bar{\lambda}=0$ then (10), (11), and (12) will collapse into

$$
\frac{d^{2} U_{s}(Y)}{d Y^{2}}=0, \quad U_{s}(0)=0, \quad U_{s}(1)=1 .
$$


Its solution is $U_{s}(Y)=Y$, which is conventional Couette flow. When $R_{e} \rightarrow-\infty, \bar{N}=0$, and $\bar{\lambda}=0$, then (13) gives $U_{s}(Y)=$ 1 , except at the bottom wall (at the bottom wall $Y=$ 0 and $\left.U_{s}(0)=0\right)$. When $R_{e} \rightarrow \infty, \bar{N}=0$, and $\bar{\lambda}=0$, then (13) gives $U_{s}(Y)=0$, except at the upper wall (at the upper wall $Y=1$ and $\left.U_{s}(1)=1\right)$. The transient part problem becomes

$$
\begin{gathered}
\frac{\partial U_{t}}{\partial T}+R_{e} \frac{\partial U_{t}}{\partial Y}=\frac{\partial^{2} U_{t}}{\partial Y^{2}}-\bar{N} U_{t}, \\
U_{t}(0, T)-\left.\bar{\lambda} \frac{\partial U_{t}(Y, T)}{\partial Y}\right|_{Y=0}=0, \\
U_{t}(1, T)+\left.\bar{\lambda} \frac{\partial U_{t}(Y, T)}{\partial Y}\right|_{Y=1}=1, \\
U_{t}(Y, 0)=-U_{s}(Y) .
\end{gathered}
$$

The solution can be derived by using Laplace transformation techniques [27]. The Laplace transform pair is defined by

$$
\begin{gathered}
\bar{U}(Y, s)=L[U(Y, T)]=\int_{0}^{\infty} U(Y, T) e^{-s T} d T, \\
U(Y, T)=L^{-1}[\bar{U}(Y, s)]=\frac{1}{2 \pi \iota} \int_{\lambda_{1}-\iota \infty}^{\lambda_{1}+\infty} \bar{U}(Y, s) e^{s T} d s,
\end{gathered}
$$

over that range of values of $s$ for which the integrals exist. Here, $s$ is a parameter, real or complex, $L$ is the operator that transforms $U(Y, T)$ into $\bar{U}(Y, s)$, called Laplace transform operator, and $L^{-1}$ is the inverse Laplace transform operator. The solution can be shown as

$$
\begin{array}{r}
U_{t}(Y, T)=L^{-1}\left[\left(e^{a Y}((1-\bar{\lambda} a) \sinh (b Y)+\bar{\lambda} b \cosh (b Y))\right)\right. \\
\times\left(e ^ { a } s \left(\left(1-\bar{\lambda}^{2}\left(a^{2}-b^{2}\right)\right) \sinh (b)\right.\right. \\
\left.+2 \bar{\lambda} b \cosh (b)))^{-1}\right]-U_{s}
\end{array}
$$

where $a=R_{e} / 2$ and $b=\sqrt{R_{e}^{2} / 4+s+\bar{N}}$. In inverse Laplace transform of the above equation we have simple pole at $s=0$ and infinite number of poles (located on the negative real axis) at $s_{n}=-\left(l_{n}^{2}+a^{2}+\bar{N}\right),(n=1,2,3, \ldots)$, where $l_{n}=i b_{n},\left(b_{n}=\sqrt{R_{e}^{2} / 4+s_{n}+\bar{N}}\right)$ and are given by

$$
\tan \left(l_{n}\right)=\frac{-2 \bar{\lambda} l_{n}}{1-\bar{\lambda}^{2}\left(a^{2}+l_{n}^{2}\right)} .
$$

The transient part velocity will be

$$
\begin{aligned}
U_{t}(Y, T)= & \sum_{n=1}^{\infty} \operatorname{Re} s\left[\bar{U}_{t}(Y, s) e^{s T}\right]_{s=s_{n}} \\
& +\operatorname{Re} s\left[\bar{U}_{t}(Y, s) e^{s T}\right]_{s=0}-U_{s},
\end{aligned}
$$

where Re $s$ stands for residue and $\bar{U}_{t}(Y, s)$ is given by

$$
\bar{U}_{t}(Y, s)=\frac{e^{a Y}((1-\bar{\lambda} a) \sinh (b Y)+\bar{\lambda} b \cosh (b Y))}{e^{a} s\left(\left(1-\bar{\lambda}^{2}\left(a^{2}-b^{2}\right)\right) \sinh (b)+2 \bar{\lambda} b \cosh (b)\right)} .
$$

We have

$$
\sum_{n=1}^{\infty} \operatorname{Re} s\left[\bar{U}_{t}(Y, s) e^{s T}\right]_{s=s_{n}}=\sum_{n=1}^{\infty} \frac{G_{1}\left(l_{n}, Y\right)}{G_{2}\left(l_{n}\right)} e^{s_{n} T},
$$

where

$$
\begin{aligned}
G_{1}\left(l_{n}, Y\right)=e^{a Y}( & (1-\bar{\lambda} a) \sin \left(l_{n} Y\right) \\
& \left.+\bar{\lambda} l_{n} \cos \left(l_{n} Y\right)\right), \\
G_{2}\left(l_{n}\right)=e^{a} s_{n}( & \bar{\lambda}(\bar{\lambda}+1) \sin \left(l_{n}\right) \\
& \left.-\left(1+2 \bar{\lambda}-\bar{\lambda}^{2}\left(a^{2}+l_{n}^{2}\right)\right) \frac{\cos \left(l_{n}\right)}{2 l_{n}}\right) .
\end{aligned}
$$

The residue at $s=0$ gives steady velocity

$$
\begin{gathered}
\operatorname{Re} s\left[\bar{U}_{t}(Y, s) e^{s T}\right]_{s=0} \\
=\lim _{s \rightarrow 0}\left[s \bar{U}_{t}(Y, s) e^{s T}\right] \\
=\lim _{s \rightarrow 0}\left(e^{a Y}((1-\bar{\lambda} a) \sinh (b Y)\right. \\
\left.+\bar{\lambda} b \cosh (b Y)) e^{s T}\right) \\
\times\left(e ^ { a } \left(\left(1-\bar{\lambda}^{2}\left(a^{2}-b^{2}\right)\right) \sinh (b)\right.\right. \\
+2 \bar{\lambda} b \cosh (b)))^{-1},
\end{gathered}
$$

$$
\lim _{s \rightarrow 0} b=\lim _{s \rightarrow 0} \sqrt{\frac{R_{e}^{2}}{4}+s+\bar{N}}=\sqrt{\frac{R_{e}^{2}}{4}+\bar{N}}=b_{1} .
$$

Therefore,

$$
\begin{gathered}
\operatorname{Res}\left[\bar{U}_{t}(Y, s) e^{s T}\right]_{s=0} \\
=\left(e^{a Y}\left[(1-\bar{\lambda} a) \sinh \left(b_{1} Y\right)+\bar{\lambda} b_{1} \cosh \left(b_{1} Y\right)\right]\right) \\
\times\left(e ^ { a } \left[\left(1-\bar{\lambda}^{2}\left(a^{2}-b_{1}^{2}\right)\right)\right.\right. \\
\left.\left.\quad \times \sinh \left(b_{1}\right)+2 \bar{\lambda} b_{1} \cosh \left(b_{1}\right)\right]\right)^{-1} .
\end{gathered}
$$

By using (13), we get

$$
\operatorname{Re} s\left[\bar{U}_{t}(Y, s) e^{s T}\right]_{s=0}=U_{s} .
$$


The transient part velocity from (24) becomes

$$
U_{t}(Y, T)=\sum_{n=1}^{\infty} \frac{G_{1}\left(l_{n}, Y\right)}{G_{2}\left(l_{n}\right)} e^{s_{n} T},
$$

$U_{t}(Y, T)$

$$
\begin{gathered}
=\sum_{n=1}^{\infty}\left[\left(e^{a Y}\left((1-\bar{\lambda} a) \sin \left(l_{n} Y\right)+\bar{\lambda} l_{n} \cos \left(l_{n} Y\right)\right)\right)\right. \\
\times\left(e ^ { a } s _ { n } \left(\bar{\lambda}(\bar{\lambda}+1) \sin \left(l_{n}\right)\right.\right. \\
-\left(1+2 \bar{\lambda}-\bar{\lambda}^{2}\left(a^{2}+l_{n}^{2}\right)\right) \\
\left.\left.\left.\times \frac{\cos \left(l_{n}\right)}{2 l_{n}}\right)\right)^{-1} e^{s_{n} T}\right]
\end{gathered}
$$

Therefore, the overall transient solution from (9) becomes

$$
\begin{gathered}
U(Y, T)=\sum_{n=1}^{\infty}\left\{\left(e^{a Y}\left((1-\bar{\lambda} a) \sin \left(l_{n} Y\right)+\bar{\lambda} l_{n} \cos \left(l_{n} Y\right)\right)\right.\right. \\
\times\left(e ^ { a } s _ { n } \left(\bar{\lambda}(\bar{\lambda}+1) \sin \left(l_{n}\right)\right.\right. \\
-\left(1+2 \bar{\lambda}-\bar{\lambda}^{2}\left(a^{2}+l_{n}^{2}\right)\right) \\
+\left(e^{a Y}\left[(1-\bar{\lambda} a) \sinh \left(b_{1} Y\right)+\bar{\lambda} b_{1} \cosh \left(b_{1} Y\right)\right]\right) \\
\times\left(e ^ { a } \left[\left(1-\bar{\lambda}^{2}\left(a^{2}-b_{1}^{2}\right)\right) \sinh \left(b_{1}\right)\right.\right. \\
\left.\left.+2 \bar{\lambda} b_{1} \cosh \left(b_{1}\right)\right]\right)^{-1} \cdot
\end{gathered}
$$

To recover Fang [1] result number (13) we substitute $\bar{\lambda}=$ $0, \bar{N}=0, a=R_{e} / 2$, and $b_{1}=R_{e} / 2$ into (32), and we get

$$
\begin{aligned}
U(Y, T)= & \sum_{n=1}^{\infty}\left\{\frac{\sin \left(l_{n} Y\right) e^{s_{n} T} e^{\left(R_{e} / 2\right) Y}}{-e^{R_{e} / 2} s_{n} \cos \left(l_{n}\right) /\left(2 l_{n}\right)}\right\} \\
& +\frac{\sinh \left(\left(R_{e} / 2\right) Y\right) e^{\left(R_{e} / 2\right) Y}}{e^{R_{e} / 2} \sinh \left(R_{e} / 2\right)}
\end{aligned}
$$

Since $\bar{\lambda}=0$, so (23) gives

$$
\begin{array}{r}
\tan \left(l_{n}\right)=0 \Longrightarrow \sin \left(l_{n}\right)=0 \Longrightarrow l_{n}=\arcsin (0)=n \pi \\
n=1,2,3, \ldots .
\end{array}
$$

Therefore $\cos \left(l_{n}\right)=\cos (n \pi)=(-1)^{n}$; also we have $l_{n}=i b_{n}$, $\left(b_{n}=\sqrt{R_{e}^{2} / 4+s_{n}+\bar{N}}\right)$, but $\bar{N}=0$, so

$$
\begin{aligned}
l_{n} & =i \sqrt{\frac{R_{e}^{2}}{4}+s_{n}} \Longrightarrow n \pi=i \sqrt{\frac{R_{e}^{2}}{4}+s_{n}} \\
& \Longrightarrow n^{2} \pi^{2}=-\left(\frac{R_{e}^{2}}{4}+s_{n}\right) \\
& \Longrightarrow s_{n}=-\left(n^{2} \pi^{2}+\frac{R_{e}^{2}}{4}\right) .
\end{aligned}
$$

Using $l_{n}=n \pi, \cos \left(l_{n}\right)=(-1)^{n}$, and $s_{n}=-\left(n^{2} \pi^{2}+R_{e}^{2} / 4\right)$ into (33) we obtain

$$
\begin{aligned}
U(Y, T)= & \frac{2 \pi e^{-\left(R_{e}^{2} / 4\right) T} e^{\left(R_{e} / 2\right) Y}}{e^{R_{e} / 2}} \\
& \times \sum_{n=1}^{\infty}\left\{\frac{n(-1)^{n} \sin (n \pi Y) e^{-n^{2} \pi^{2} T}}{\left(n^{2} \pi^{2}+R_{e}^{2} / 4\right)}\right\} \\
& +\frac{\sinh \left(\left(R_{e} / 2\right) Y\right) e^{\left(R_{e} / 2\right) Y}}{e^{R_{e} / 2} \sinh \left(R_{e} / 2\right)} .
\end{aligned}
$$

Consider

$$
\begin{aligned}
\frac{\sinh \left(\left(R_{e} / 2\right) Y\right) e^{\left(R_{e} / 2\right) Y}}{e^{R_{e} / 2} \sinh \left(R_{e} / 2\right)} \\
=\frac{e^{\left(R_{e} / 2\right) Y}\left(e^{\left(R_{e} / 2\right) Y}-e^{-\left(R_{e} / 2\right) Y}\right)}{e^{R_{e} / 2}\left(e^{R_{e} / 2}-e^{-R_{e} / 2}\right)} \\
=\frac{\left(e^{R_{e} Y}-1\right)}{\left(e^{R_{e}}-1\right)}=\frac{\left(1-e^{R_{e} Y}\right)}{\left(1-e^{R_{e}}\right)} .
\end{aligned}
$$

So (36) becomes

$$
\begin{aligned}
U(Y, T)= & \frac{2 \pi e^{-\left(R_{e}^{2} / 4\right) T} e^{\left(R_{e} / 2\right) Y}}{e^{R_{e} / 2}} \\
& \times \sum_{n=1}^{\infty}\left\{\frac{n(-1)^{n} \sin (n \pi Y) e^{-n^{2} \pi^{2} T}}{\left(n^{2} \pi^{2}+R_{e}^{2} / 4\right)}\right\} \\
& +\frac{\left(1-e^{R_{e} Y}\right)}{\left(1-e^{R_{e}}\right)} .
\end{aligned}
$$

Result number (13) of Fang [1] is exactly followed in (38).

2.2. Steady State Temperature. It is too difficult to exactly solve the transient energy equation with viscous dissipation for this problem by using $U(Y, T)$. We solve the steady state energy equation, which in dimensionless form is given by [1]

$$
\begin{gathered}
P_{r} R_{e} \frac{d \sigma}{d Y}=\frac{d^{2} \sigma}{d Y^{2}}+E_{c} P_{r}\left(\frac{d U_{s}}{d Y}\right)^{2}, \\
\sigma(0)=0, \quad \sigma(1)=1,
\end{gathered}
$$


where $P_{r}=\nu / \alpha$ is the Prandtl number, $E_{c}=U_{0}^{2} / c_{p}\left(\theta_{1}-\right.$ $\left.\theta_{2}\right)$ is the Eckert number, $\theta_{1}$ is temperature of the bottom fixed plate, $\theta_{2}$ is temperature of the top moving plate, $\sigma$ is dimensionless temperature $\left(\sigma=\left(\theta-\theta_{1}\right) /\left(\theta_{2}-\theta_{1}\right)\right)$, and $U_{s}$ is the steady state velocity given by (13). The solution of (39) with BCs (35) for $P_{r} \neq 1$, is

$$
\begin{aligned}
& \sigma(Y)=\frac{\left(e^{P_{r} R_{e} Y}-1\right)}{\left(e^{P_{r} R_{e}}-1\right)} \\
&+E_{c} P_{r} M\left[\frac{\left(e^{P_{r} R_{e} Y}-1\right)}{\left(e^{P_{r} R_{e}}-1\right)}\right. \\
& \times\left\{N_{1}\left(e^{2\left(a+b_{1}\right)}-1\right)\right. \\
&\left.+N_{2}\left(e^{2\left(a-b_{1}\right)}-1\right)-N_{3}\left(e^{2 a}-1\right)\right\} \\
&+\left\{N_{1}\left(1-e^{2\left(a+b_{1}\right) Y}\right)\right. \\
&\left.\left.+N_{2}\left(1-e^{2\left(a-b_{1}\right) Y}\right)-N_{3}\left(1-e^{2 a Y}\right)\right\}\right],
\end{aligned}
$$

where

$$
\begin{gathered}
N_{1}=\frac{M_{1}}{L_{1}}, \quad N_{2}=\frac{M_{2}}{L_{2}}, \quad N_{3}=\frac{M_{3}}{L_{3}}, \\
L_{1}=4\left(a+b_{1}\right)^{2}-2 P_{r} R_{e}\left(a+b_{1}\right), \\
M=\frac{4\left(a-b_{1}\right)^{2}-2 P_{r} R_{e}\left(a-b_{1}\right), \quad L_{3}=R_{e}^{2}\left(1-P_{r}\right),}{\left[e^{a}\left\{\left(1-\bar{\lambda}^{2}\left(a^{2}-b_{1}^{2}\right)\right) \sinh \left(b_{1}\right)+2 \bar{\lambda} b_{1} \cosh \left(b_{1}\right)\right\}\right]^{2}}, \\
M_{1}=\left(\frac{B_{1}+b_{1}}{2}\right)^{2}, \quad M_{2}=\left(\frac{B_{1}-b_{1}}{2}\right)^{2}, \\
M_{3}=\left(\frac{B_{1}^{2}-b_{1}^{2}}{2}\right), \\
B_{1}=\left(a-\bar{\lambda}\left(a^{2}-b_{1}^{2}\right)\right) .
\end{gathered}
$$

When viscous dissipation term is negligible, then energy equation (39) is

$$
\frac{d^{2} \sigma}{d Y^{2}}-P_{r} R_{e} \frac{d \sigma}{d Y}=0, \quad \sigma(0)=0, \quad \sigma(1)=1 .
$$

Its solution is

$$
\sigma(Y)=\frac{\left(e^{P_{r} R_{e} Y}-1\right)}{\left(e^{P_{r} R_{e}}-1\right)} .
$$

So the first term in (40) is the solution of energy equation when viscous dissipation term is negligible and the second term in (40) is the temperature profile from viscous dissipation and MHD. It is found from (40) that the temperature profile is linearly dependent upon the Eckert number. The Nusselt number at the walls will be $N_{u}=|d \sigma / d Y|_{w}$. The Nusselt number for the bottom wall, $Y=0$, is

$$
\begin{aligned}
N_{u_{1}}=\left|\frac{d \sigma}{d Y}\right|_{Y=0}, & \\
N_{u_{1}}=\mid \frac{P_{r} R_{e}}{\left(e^{P_{r} R_{e}}-1\right)}\left[1+E_{c} P_{r} M\{\right. & N_{1}\left(e^{2\left(a+b_{1}\right)}-1\right) \\
& +N_{2}\left(e^{2\left(a-b_{1}\right)}-1\right) \\
& \left.\left.-N_{3}\left(e^{2 a}-1\right)\right\}\right] \\
-2 E_{c} P_{r} M\left\{N_{1}\left(a+b_{1}\right)\right. & \\
+ & \left.+N_{2}\left(a-b_{1}\right)-N_{3} a\right\} \mid .
\end{aligned}
$$

The Nusselt number for the upper wall, $Y=1$, is

$$
\begin{aligned}
& N_{u_{2}}=\left|\frac{d \sigma}{d Y}\right|_{Y=1}, \\
& N_{u_{2}}=\mid \frac{P_{r} R_{e} e^{P_{r} R_{e}}}{\left(e^{P_{r} R_{e}}-1\right)} \\
& \times\left[1+E_{c} P_{r} M\left\{N_{1}\left(e^{2\left(a+b_{1}\right)}-1\right)\right.\right. \\
& +N_{2}\left(e^{2\left(a-b_{1}\right)}-1\right) \\
& \left.\left.-N_{3}\left(e^{2 a}-1\right)\right\}\right] \\
& -2 E_{c} P_{r} M\left\{N_{1}\left(a+b_{1}\right) e^{2\left(a+b_{1}\right)}\right. \\
& \left.+N_{2}\left(a-b_{1}\right) e^{2\left(a-b_{1}\right)}-N_{3} a e^{2 a}\right\} \mid .
\end{aligned}
$$

When $P_{r}=1$, then the energy equation is

$$
\begin{gathered}
\frac{d^{2} \sigma}{d Y^{2}}-R_{e} \frac{d \sigma}{d Y}=-E_{c} M\left\{M_{1} e^{2\left(a+b_{1}\right) Y}\right. \\
\left.+M_{2} e^{2\left(a-b_{1}\right) Y}-M_{3} e^{2 a Y}\right\}, \\
\sigma(0)=0, \quad \sigma(1)=1 .
\end{gathered}
$$

The solution of (46) is

$$
\begin{array}{r}
\sigma(Y)=\frac{\left(e^{R_{e} Y}-1\right)}{\left(e^{R_{e}}-1\right)}\left[1+E_{c} M\left\{N_{4}\left(e^{2\left(a+b_{1}\right)}-1\right)\right.\right. \\
+N_{5}\left(e^{2\left(a-b_{1}\right)}-1\right) \\
\left.\left.-\frac{M_{3}}{R_{e}} e^{2 a}\right\}\right] \\
+2 E_{c} M\left\{N_{4}\left(1-e^{2\left(a+b_{1}\right) Y}\right)\right. \\
\left.+N_{5}\left(1-e^{2\left(a-b_{1}\right) Y}\right)+\frac{M_{3} Y}{R_{e}} e^{2 a Y}\right\},
\end{array}
$$


where

$$
\begin{gathered}
N_{4}=\frac{M_{1}}{L_{4}}, \quad N_{5}=\frac{M_{2}}{L_{5}}, \\
L_{4}=4\left(a+b_{1}\right)^{2}-2 R_{e}\left(a+b_{1}\right), \\
L_{5}=4\left(a-b_{1}\right)^{2}-2 R_{e}\left(a-b_{1}\right) .
\end{gathered}
$$

The Nusselt number in this case is

$$
\begin{aligned}
& N_{u_{1}}=\mid \frac{R_{e}}{\left(e^{R_{e}}-1\right)} \\
& \times\left[1+E_{c} M\left\{N_{4}\left(e^{2\left(a+b_{1}\right)}-1\right)\right.\right. \\
& +N_{5}\left(e^{2\left(a-b_{1}\right)}-1\right) \\
& \left.\left.-\frac{M_{3}}{R_{e}} e^{2 a}\right\}\right] \\
& -2 E_{c} M\left\{N_{4}\left(a+b_{1}\right)\right. \\
& \left.+N_{5}\left(a-b_{1}\right)-\frac{M_{3}}{R_{e}}\right\} \mid \\
& N_{u_{2}}=\mid \frac{R_{e} e^{R_{e}}}{\left(e^{R_{e}}-1\right)} \\
& \times\left[1+E_{c} M\left\{N_{4}\left(e^{2\left(a+b_{1}\right)}-1\right)\right.\right. \\
& +N_{5}\left(e^{2\left(a-b_{1}\right)}-1\right) \\
& \left.\left.-\frac{M_{3}}{R_{e}} e^{2 a}\right\}\right] \\
& -2 E_{c} M\left\{N_{4}\left(a+b_{1}\right) e^{2\left(a+b_{1}\right)}\right. \\
& \left.+N_{5}\left(a-b_{1}\right) e^{2\left(a-b_{1}\right)}-\frac{M_{3}}{R_{e}} a e^{2 a}\right\} \mid .
\end{aligned}
$$

It is worth recalling in the vicinity of (40) that when $\bar{\lambda}=$ $0, \bar{N}=0$, then result number (18) of Fang [1] can be derived.

\section{Graphs and Discussion}

In this part we discuss the variation of the transient part velocity $U_{t}$, overall transient velocity $U$, and steady state velocity $U_{s}$ with distance from the wall $Y$ for different values of Reynolds number $R_{e}$, magnetic field parameter $\bar{N}$, slip parameter $\bar{\lambda}$, and time $T$.

Figures 1 and 2 show the variation of transient part velocity $U_{t}$ with distance from the wall $Y$ for several values of magnetic parameter $\bar{N}$, by keeping $\bar{\lambda}$ and $T$ fixed. Figure 1 shows that when there is mass suction $R_{e}<0$ at the

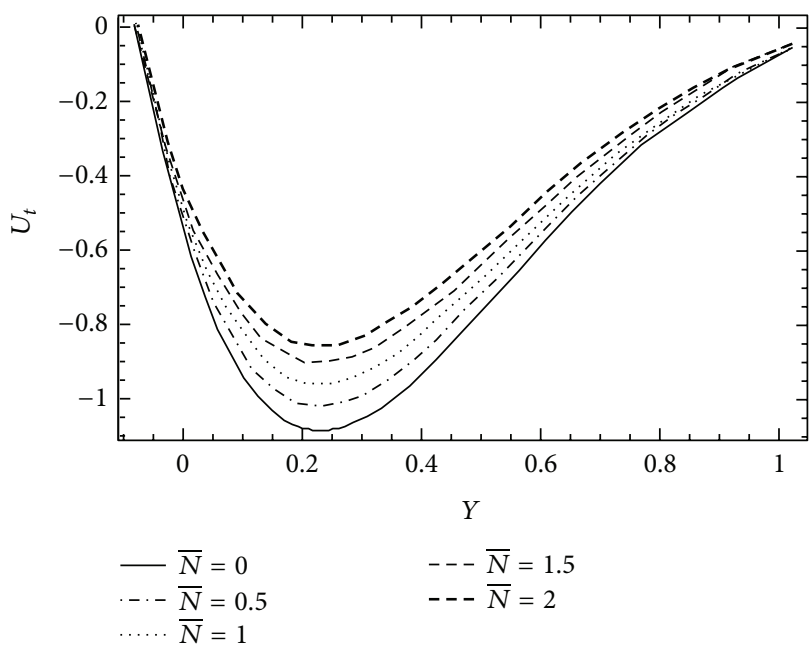

FIgURE 1: Variation of $U_{t}$ with $Y$ for several values of $\bar{N}$ with $R_{e}=$ $-5, \bar{\lambda}=0.1$, and $T=0.05$.

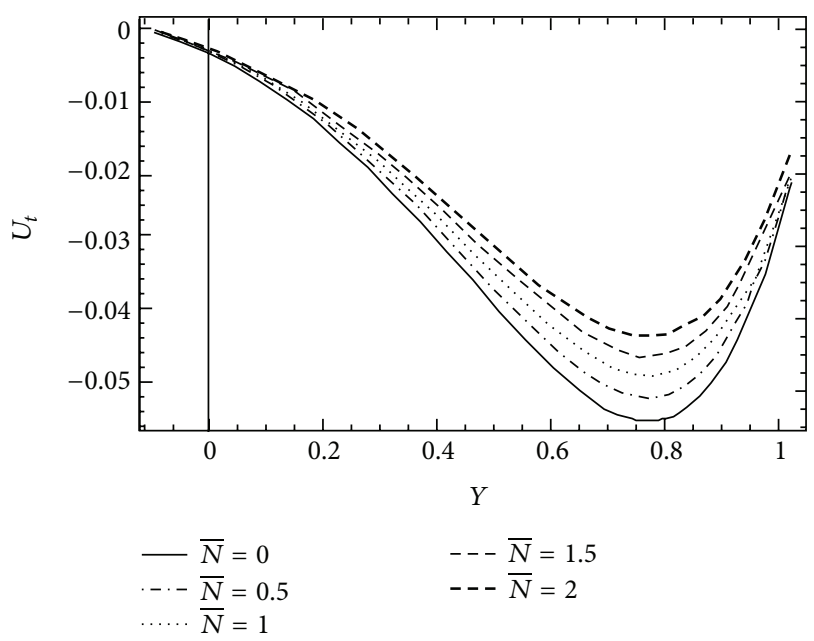

FIgURE 2: Variation of $U_{t}$ with $Y$ for several values of $\bar{N}$ with $R_{e}=$ $5, \bar{\lambda}=0.1$, and $T=0.05$.

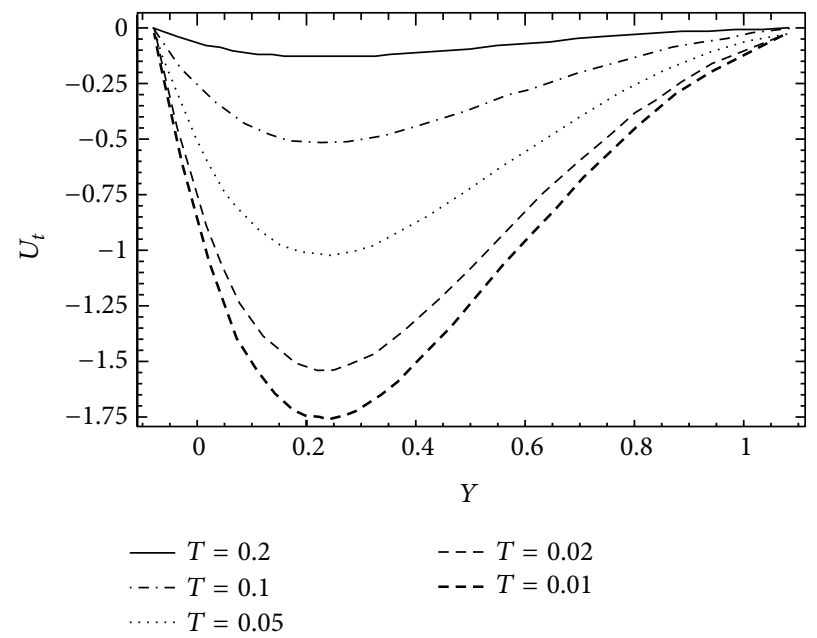

FIGURE 3: Variation of $U_{t}$ with $Y$ for several values of $T$ with $R_{e}=$ $-5, \bar{\lambda}=0.1$, and $\bar{N}=0.5$. 


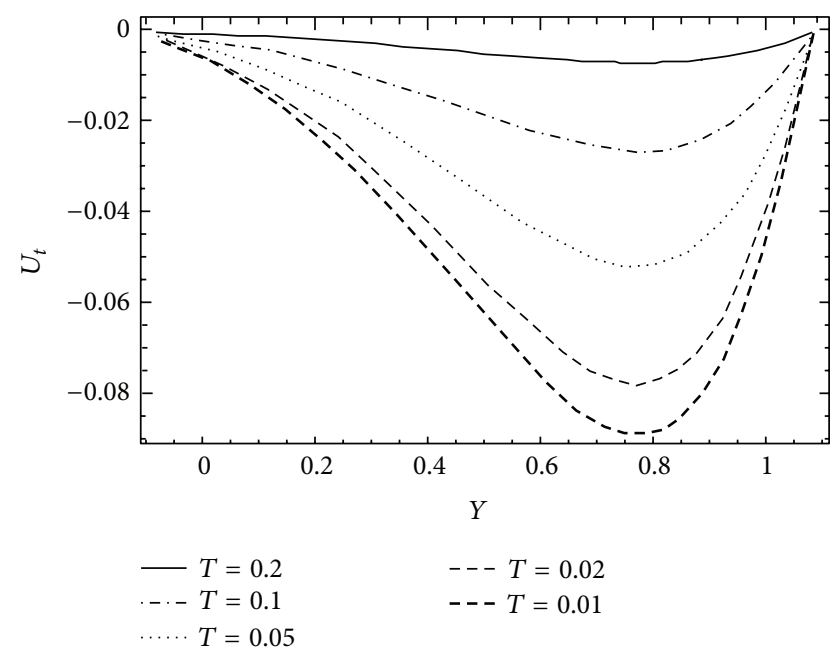

FIGURE 4: Variation of $U_{t}$ with $Y$ for several values of $T$ with $R_{e}=$ $5, \bar{\lambda}=0.1$, and $\bar{N}=0.5$.

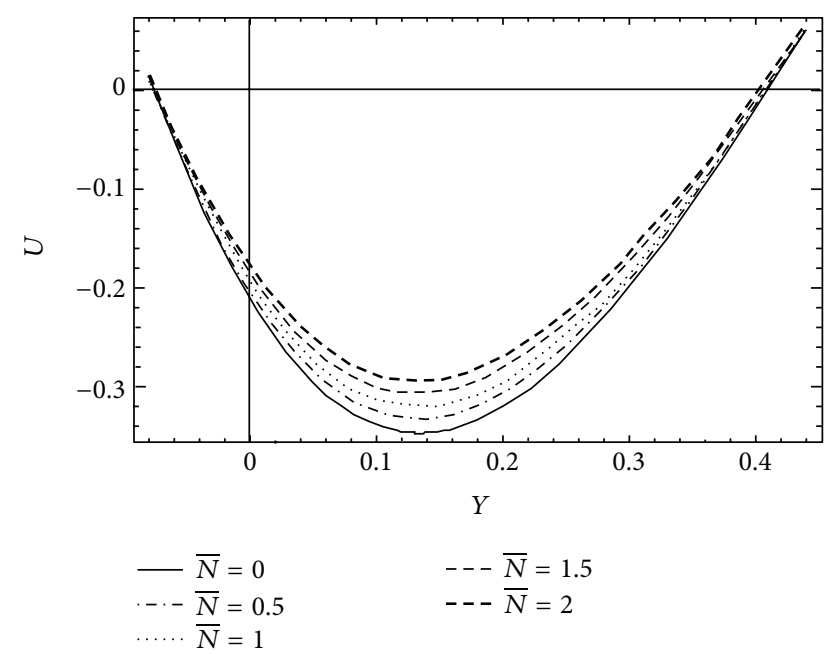

FIGURE 5: Variation of $U$ with $Y$ for several values of $\bar{N}$ with $R_{e}=$ $-5, \bar{\lambda}=0.1$, and $T=0.05$.

top wall, with increase in magnetic field, transient part velocity decreases in magnitude. Figure 2 shows that when there is mass injection $R_{e}>0$ at the bottom wall, with increase in magnetic field, transient part velocity $U_{t}$ decreases and will become weaker as compared to the case of suction. From Figure 3 it is observed that for suction at top wall and for fixed values of $\bar{\lambda}, \bar{N}$ transient part velocity $U_{t}$ decreases in magnitude with increase in time. Figure 4 shows that for injection at bottom wall and for fixed values of $\bar{\lambda}, \bar{N}$ transient part velocity $U_{t}$ decreases in magnitude with increase in time. From Figures 3 and 4 it is seen that the transient part velocity will decay with time, which is consistent with what we expected. From Figures 3 and 4 it is clear that, after a certain time, the transient part velocity will die away and velocity will become developed. Figures 5 and 6 indicate variation of overall transient velocity $U$ with $Y$ for fixed values of $\bar{\lambda}$ and $T$. Figure 5 shows that, for $R_{e}=-5<0$,

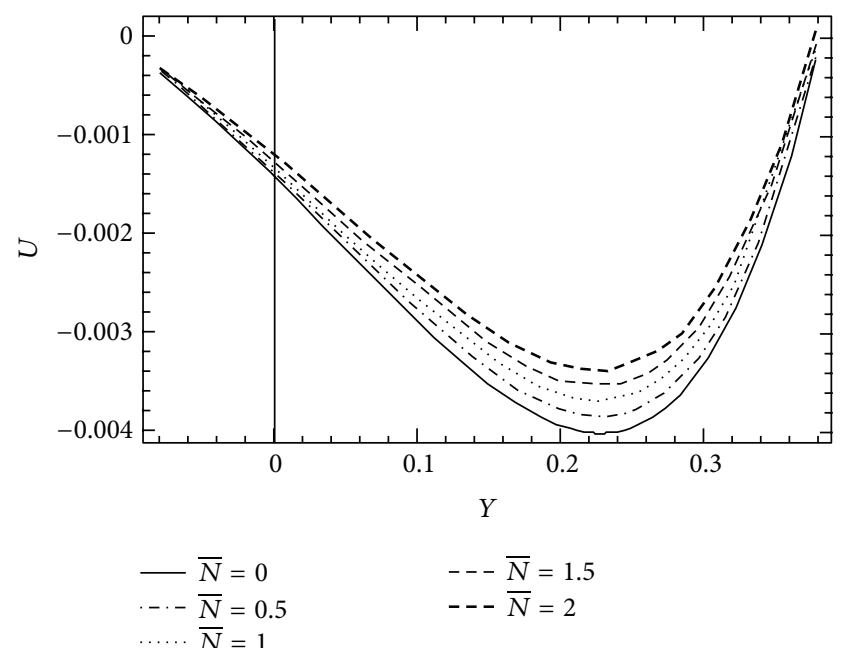

Figure 6: Variation of $U$ with $Y$ for several values of $\bar{N}$ with $R_{e}=$ $5, \bar{\lambda}=0.1$, and $T=0.05$.

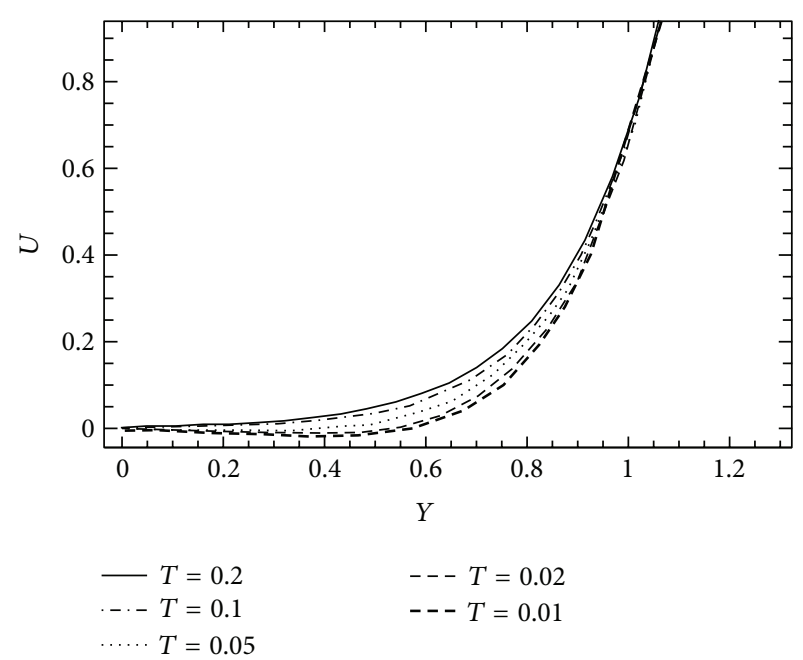

FIGURE 7: Variation of $U$ with $Y$ for several values of $T$ with $R_{e}=$ $5, \bar{\lambda}=0.1$, and $\bar{N}=0.5$.

with increase in magnetic parameter $\bar{N}$, overall transient velocity $U$ decreases. Figure 6 shows that, for $R_{e}=5>$ 0 , with increase in magnetic parameter $\bar{N}$, overall transient velocity $U$ decreases but weaker. The overall transient velocities for $R_{e}=5$ at different times are depicted in Figure 7. Figure 7 shows that for mass injection at the bottom wall, overall transient velocity $U$ increases with time.

Figures 8 and 9 illustrate the variation of steady state velocity $U_{s}$ with $Y$ for several values of slip parameter $\bar{\lambda}$ and for fixed value of $\bar{N}$. Figure 8 shows that, for suction at the top wall with increase in slip parameter $\bar{\lambda}$, steady state velocity $U_{s}$ increases. Figure 9 shows that, for injection at the bottom wall with increase in slip parameter $\bar{\lambda}$, steady state velocity $U_{s}$ decreases.

Now we discuss the variation of the steady state temperature distribution $\sigma$ with distance from the surface $Y$ for 


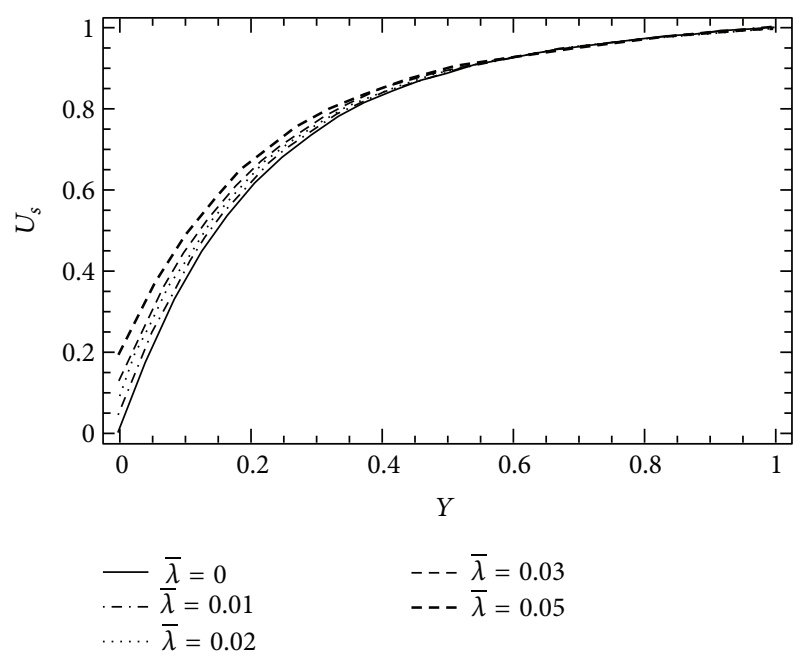

FIGURE 8: Variation of $U_{s}$ with $Y$ for several values of $\bar{\lambda}$ with $R_{e}=$ $-5, \bar{N}=0.5$.

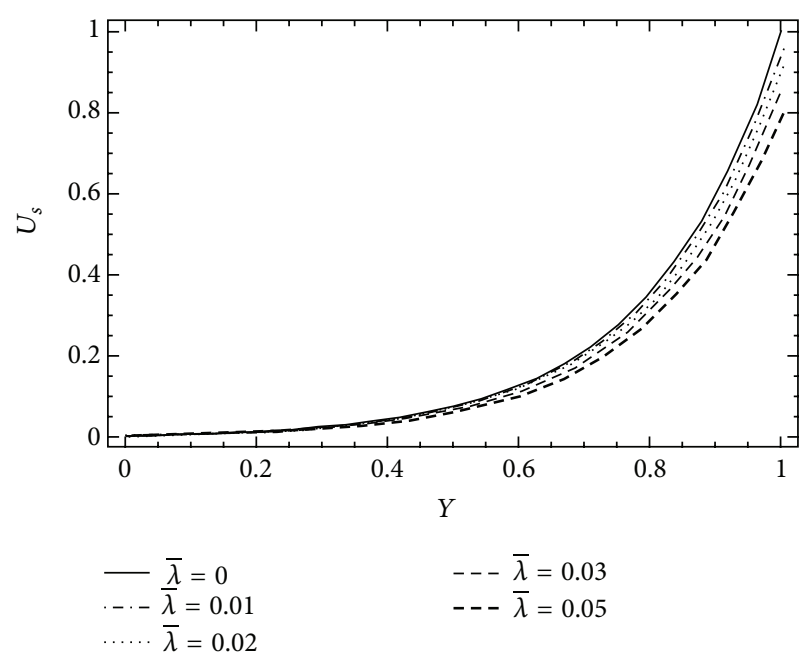

FIGURE 9: Variation of $U_{s}$ with $Y$ for several values of $\bar{\lambda}$ with $R_{e}=$ $5, \bar{N}=0.5$.

different values of five dimensionless parameters: Reynolds number $R_{e}$, Prandtl number $P_{r}$, Eckert number $E_{c}$, slip parameter $\bar{\lambda}$, and magnetic parameter $\bar{N}$. Variation of the Nusselt number at walls for different Prandtl numbers is also discussed. Figures 10 and 11 elucidate the variation of steady state temperature distribution $\sigma$ with $Y$ for several values of $P_{r}$ and for fixed values of $E_{c}, \bar{\lambda}$, and $\bar{N}$. Figure 10 shows that for $R_{e}=5>0$, (injection) temperature $\sigma$ at a point decreases with increase in $P_{r}$. Figure 11 shows that, for $R_{e}=$ $-5<0$, (suction) temperature $\sigma$ with increase in $P_{r}$ increases at a point. Comparison of Figures 10 and 11 shows that, for mass suction at the bottom wall, the maximum temperature will exist in the fluid because of viscous dissipation.

Figures 12 and 13 illustrate the variation of Nusselt numbers at the bottom wall with $R_{e}$, for fixed values of $E_{c}, \bar{\lambda}$, and $\bar{N}$ and for several values of $P_{r}$. Figure 12 shows that

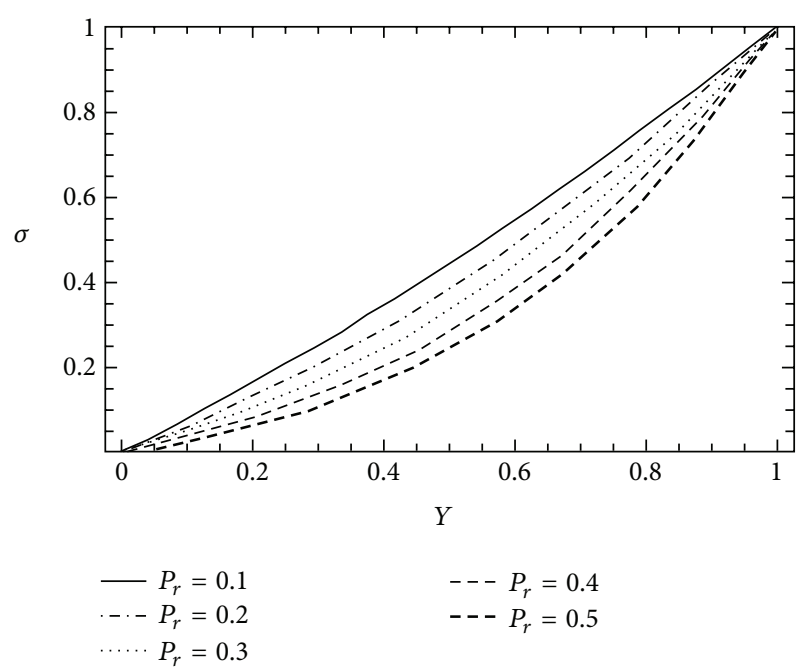

FIgURE 10: Variation of $\sigma$ with $Y$ for several values of $P_{r}$ with $R_{e}=$ $5, E_{c}=2, \bar{N}=0.5$, and $\bar{\lambda}=0.1$.

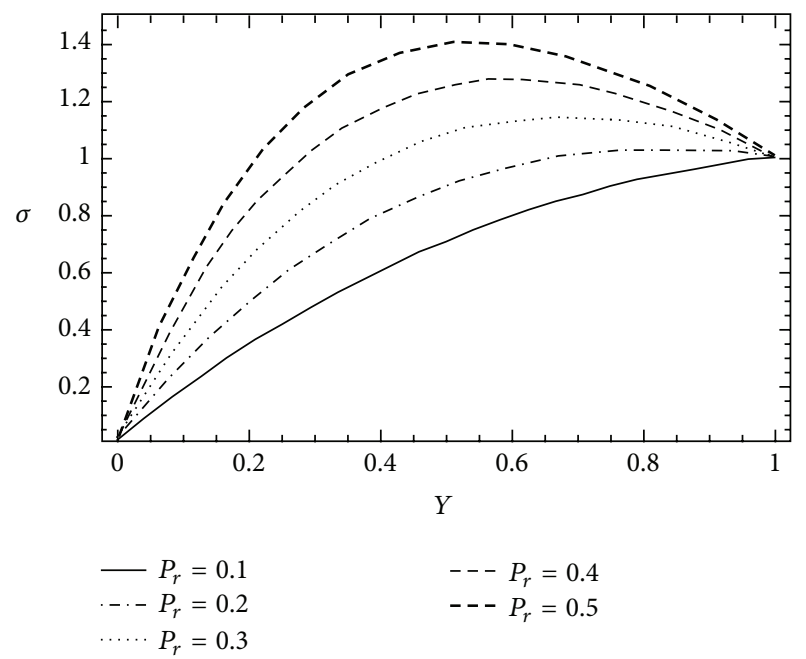

FIGURE 11: Variation of $\sigma$ with $Y$ for several values of $P_{r}$ with $R_{e}=$ $-5, E_{c}=2, \bar{N}=0.5$, and $\bar{\lambda}=0.1$.

when there is mass suction at the bottom wall, heat transfer rate $N_{u_{1}}$ increases with increase in $P_{r}$. Figure 13 shows that when there is mass injection at the bottom wall, heat transfer rate $N_{u_{1}}$ decreases with increase in $P_{r}$. Figures 14 and 15 depict the variation of Nusselt numbers at the top wall with $R_{e}$, for fixed values of $E_{c}, \bar{\lambda}$, and $\bar{N}$ and for several values of $P_{r}$. Figure 14 shows that when there is mass suction at the top wall, heat transfer rate $N_{u_{2}}$ increases in magnitude with increase in $P_{r}$. Figure 15 shows that when there is mass injection at the top wall, heat transfer rate $N_{u_{2}}$ increases with increase in $P_{r}$.

\section{Final Remarks}

In this study exact solutions for the velocity field and temperature field in the presence of magnetic field, porosity, 


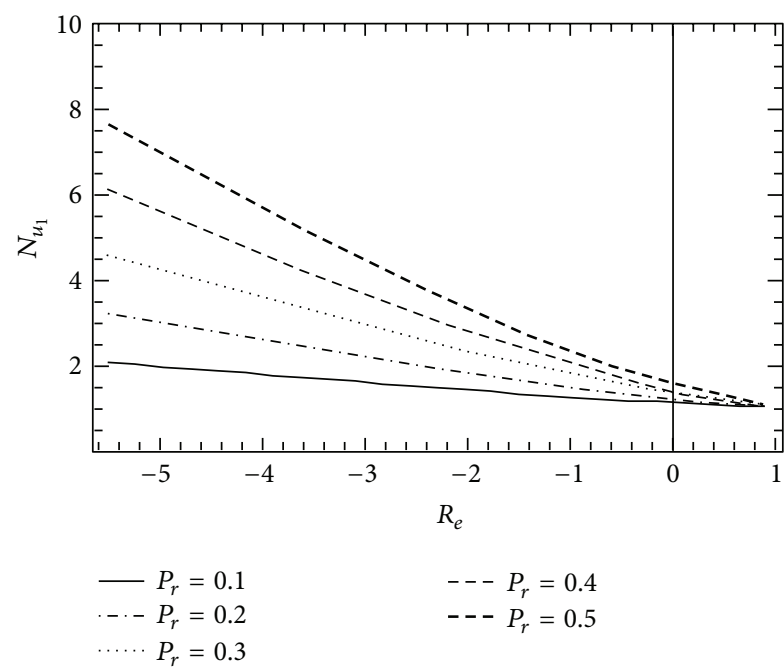

Figure 12: Variation of $N_{u_{1}}$ with $R_{e}:-5.5 \rightarrow 0.9$ for several values of $P_{r}$ with $E_{c}=2, \bar{N}=0.5$, and $\bar{\lambda}=0.1$.

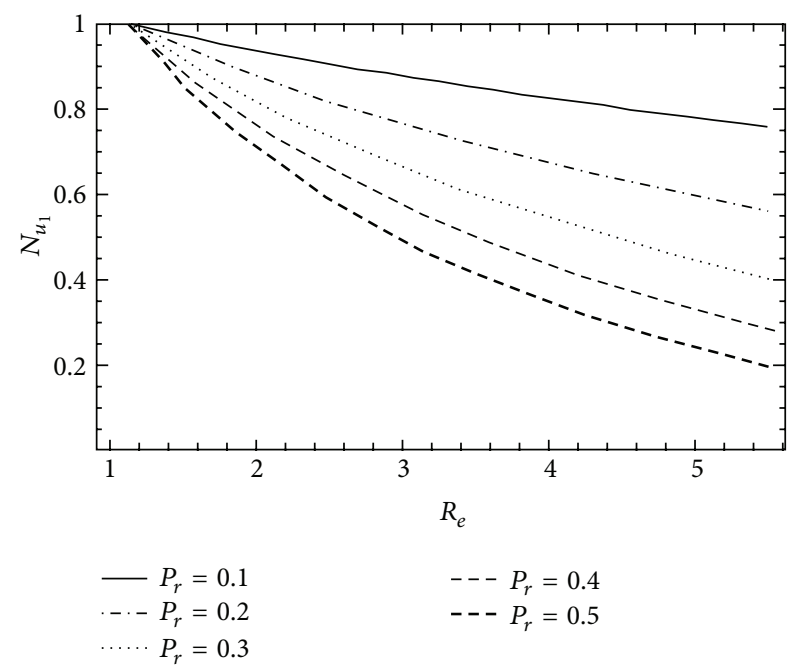

FigURE 13: Variation of $N_{u_{1}}$ with $R_{e}: 1 \rightarrow 5.5$ for several values of $P_{r}$ with $E_{c}=2, \bar{N}=0.5$, and $\bar{\lambda}=0.1$.

and slip parameter are constructed. A uniform magnetic field is applied transversely to the flow. The expressions of the velocity field and temperature field for flow subjected to the slip conditions between the two parallel plates and fluid are obtained by means of Laplace transform. The so-obtained solutions, depending on the initial and the boundary conditions, are presented as sum of the steady state transient solutions. The results of Fang [1] are also recovered by taking $\bar{\lambda}=0, \bar{N}=0$. Graphical results for mass transfer reveal that it has significant influence on the velocity distribution, temperature distribution, and heat transfer rate at the walls. The current analysis will be useful in dealing with real engineering problems.

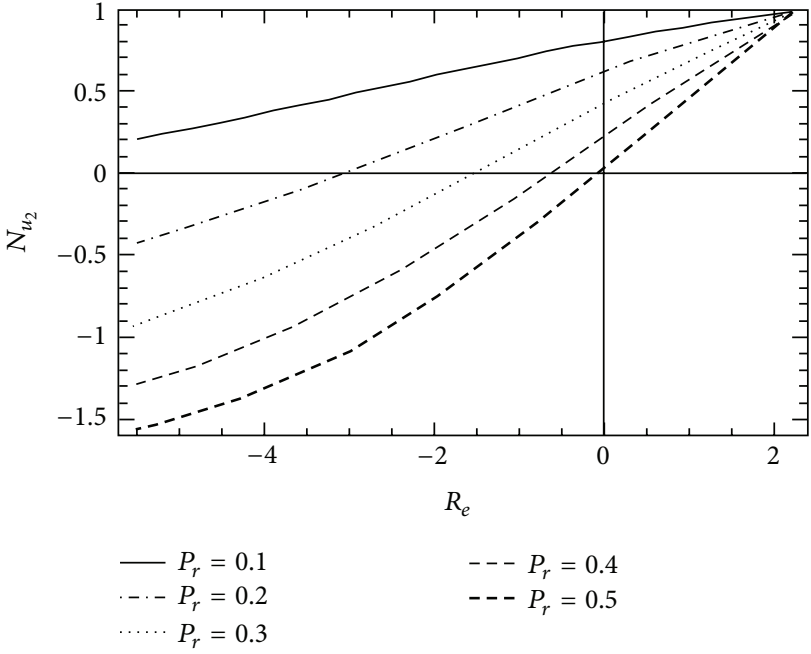

Figure 14: Variation of $N_{u_{2}}$ with $R_{e}:-5.5 \rightarrow 2.2$ for several values of $P_{r}$ with $E_{c}=2, \bar{N}=0.5$, and $\bar{\lambda}=0.1$.

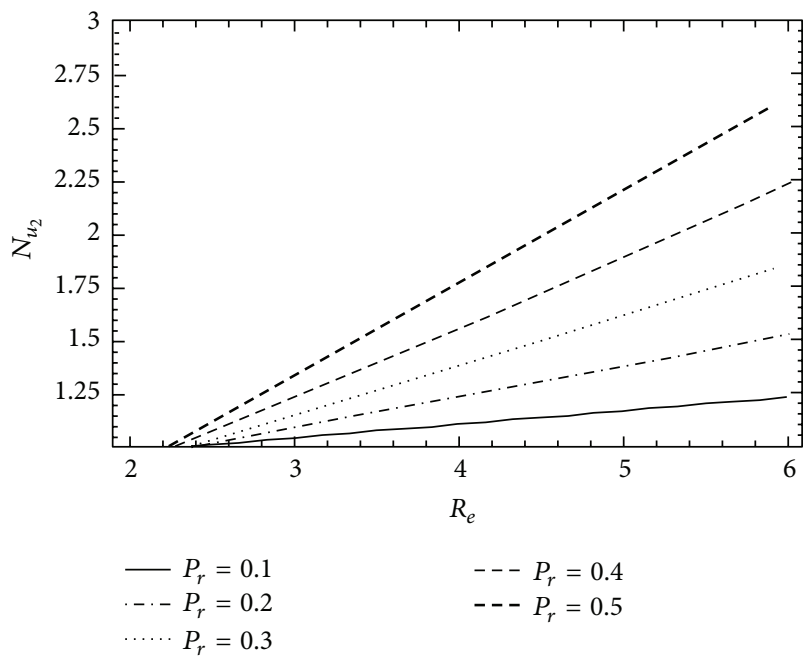

FIGURE 15: Variation of $N_{u_{2}}$ with $R_{e}: 2 \rightarrow 6$ for several values of $P_{r}$ with $E_{c}=2, \bar{N}=0.5$, and $\bar{\lambda}=0.1$.

\section{Conflict of Interests}

The authors declare that there is no conflict of interests regarding the publication of this paper. The authors do not have a direct financial relation with the commercial identity mentioned in the paper that might lead to a conflict of interests for any of them.

\section{References}

[1] T. Fang, "A note on the incompressible couette flow with porous walls," International Communications in Heat and Mass Transfer, vol. 31, no. 1, pp. 31-41, 2004.

[2] H. Schlichting and K. Gersten, Boundary Layer Theory, Springer, Berlin, Germany, 8th edition, 2000. 
[3] A. R. A. Khaled and K. Vafai, "The effect of the slip condition on Stokes and Couette flows due to an oscillating wall: exact solutions," International Journal of Non-Linear Mechanics, vol. 39, no. 5, pp. 795-809, 2004.

[4] T. Hayat, M. Khan, A. M. Siddiqui, and S. Asghar, "Transient flows of a second grade fluid," International Journal of NonLinear Mechanics, vol. 39, no. 10, pp. 1621-1633, 2004.

[5] M. Ahmad, H. Zaman, and N. Rehman, "Effects of Hall current on unsteady MHD flows of a second grade fluid," Central European Journal of Physics, vol. 8, no. 3, pp. 422-431, 2010.

[6] M. E. Erdogan, "Note on an unsteady flow of a viscous fluid due to an oscillating plane wall," International Journal of Non-Linear Mechanics, vol. 35, no. 1, pp. 1-6, 2000.

[7] K. R. Crammer and S. I. Pai, Magnetofluid Dynamics for Engineers and Applied Physicists, McGraw-Hill, New York, NY, USA, 1973.

[8] M. Ayub, H. Zaman, and M. Ahmad, "Series solution of hydromagnetic flow and heat transfer with Hall effect in a second grade fluid over a stretching sheet," Central European Journal of Physics, vol. 8, no. 1, pp. 135-149, 2010.

[9] T. Hayat, T. Javed, and Z. Abbas, "MHD flow of a micropolar fluid near a stagnation-point towards a non-linear stretching surface," Nonlinear Analysis: Real World Applications, vol. 10, no. 3, pp. 1514-1526, 2009.

[10] T. Hayat, Z. Abbas, and T. Javed, "MHD stagnation point flow and heat transfer over a permeable surface through a porous space," Journal of Porous Media, vol. 12, no. 2, pp. 183-195, 2009.

[11] S. Asghar, M. Khan, and T. Hayat, "Magnetohydrodynamic transient flows of a non-newtonian fluid," International Journal of Non-Linear Mechanics, vol. 40, no. 5, pp. 589-601, 2005.

[12] M. Khan, S. Hyder Ali, T. Hayat, and C. Fetecau, "MHD flows of a second grade fluid between two side walls perpendicular to a plate through a porous medium," International Journal of Non-Linear Mechanics, vol. 43, no. 4, pp. 302-319, 2008.

[13] M. Khan, S. Asghar, and T. Hayat, "Hall effect on the pipe flow of a Burgers' fluid: an exact solution," Nonlinear Analysis: Real World Applications, vol. 10, no. 2, pp. 974-979, 2009.

[14] R. Cortell, "MHD flow and mass transfer of an electrically conducting fluid of second grade in a porous medium over a stretching sheet with chemically reactive species," Chemical Engineering and Processing, vol. 46, no. 8, pp. 721-728, 2007.

[15] T. Hayat, H. Zaman, and M. Ayub, "Analytical solution of hydromagnetic flow with Hall effect over a surface stretching with a power-law velocity," Numerical Methods for Partial Differential Equations, vol. 27, no. 4, pp. 937-959, 2011.

[16] F. Soltani and Ü. Yilmazer, "Slip velocity and slip layer thickness in flow of concentrated suspensions," Journal of Applied Polymer Science, vol. 70, no. 3, pp. 515-522, 1998.

[17] C. Derek, D. C. Tretheway, and C. D. Meinhart, "Apparent fluid slip at hydrophobic microchannel walls," Physics of Fluids, vol. 14, no. 3, pp. L9-L12, 2002.

[18] S. Yu and T. A. Ameel, "Slip-flow heat transfer in rectangular microchannels," International Journal of Heat and Mass Transfer, vol. 44, no. 22, pp. 4225-4234, 2001.

[19] M. Ayub and H. Zaman, "Analytical solution of orthogonal flow impinging on a wall with suction or blowing," Journal of Basic \& Applied Sciences, vol. 6, no. 2, pp. 93-97, 2010.

[20] M. Khan, Z. Abbas, and T. Hayat, "Analytic solution for flow of Sisko fluid through a porous medium," Transport in Porous Media, vol. 71, no. 1, pp. 23-37, 2008.
[21] P. D. Ariel, T. Hayat, and S. Asghar, "The flow of an elasticoviscous fluid past a stretching sheet with partial slip," Acta Mechanica, vol. 187, no. 1-4, pp. 29-35, 2006.

[22] A. Raptis, C. Perdikis, and G. Tzivanidis, "Free convection flow through a porous medium bounded by a vertical surface," Journal of Physics D, vol. 14, no. 7, article 001, pp. L99-L102, 1981.

[23] A. Raptis, G. Tzivanidis, and N. Kafousias, "Free convection and mass transfer flow through a porous medium bounded by an infinite vertical limiting surface with constant suction," Letters in Heat and Mass Transfer, vol. 8, no. 5, pp. 417-424, 1981.

[24] A. Raptis, N. Kafousios, and C. Massalas, "Free convection and mass transfer flow through a porous medium bounded by an infinite vertical porous plate with constant heat flux," Zeitschrift für Angewandte Mathematik und Mechanik, vol. 62, pp. 489491, 1982.

[25] A. A. Raptis, "Unsteady free convective flow through a porous medium," International Journal of Engineering Science, vol. 21, no. 4, pp. 345-348, 1983.

[26] R. Penton, "The transient for Stokes oscillating plane: a solution in terms of tabulated functions," Journal of Fluid Mechanics, vol. 31, pp. 819-825, 1968.

[27] G. E. Roberts and H. Kaufman, Tables of Laplace Transforms, WB Saunders, Philadelphia, Pa, USA, 1966. 


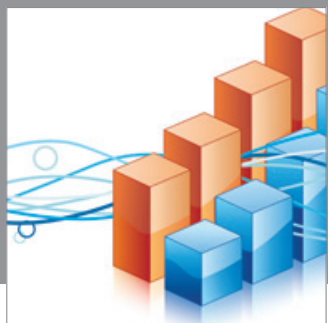

Advances in

Operations Research

mansans

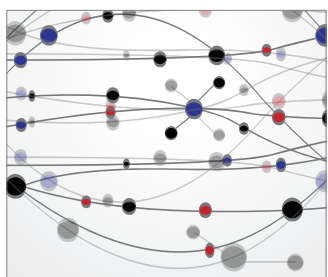

The Scientific World Journal
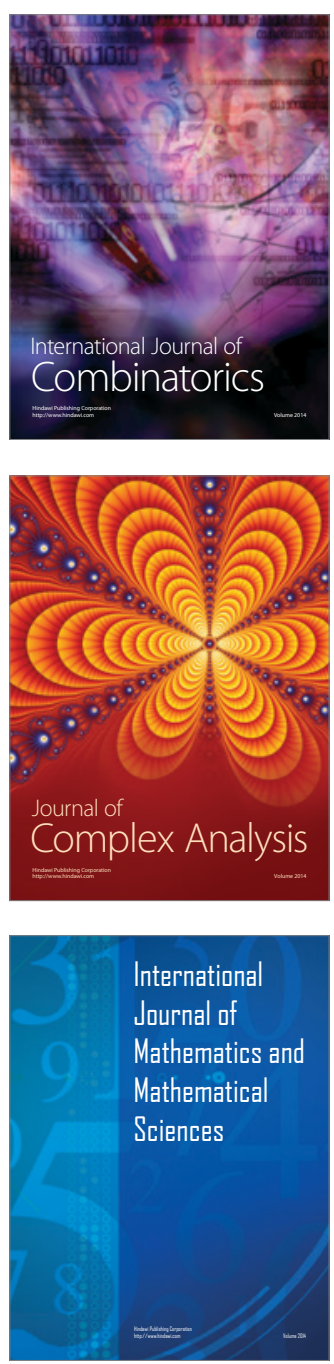
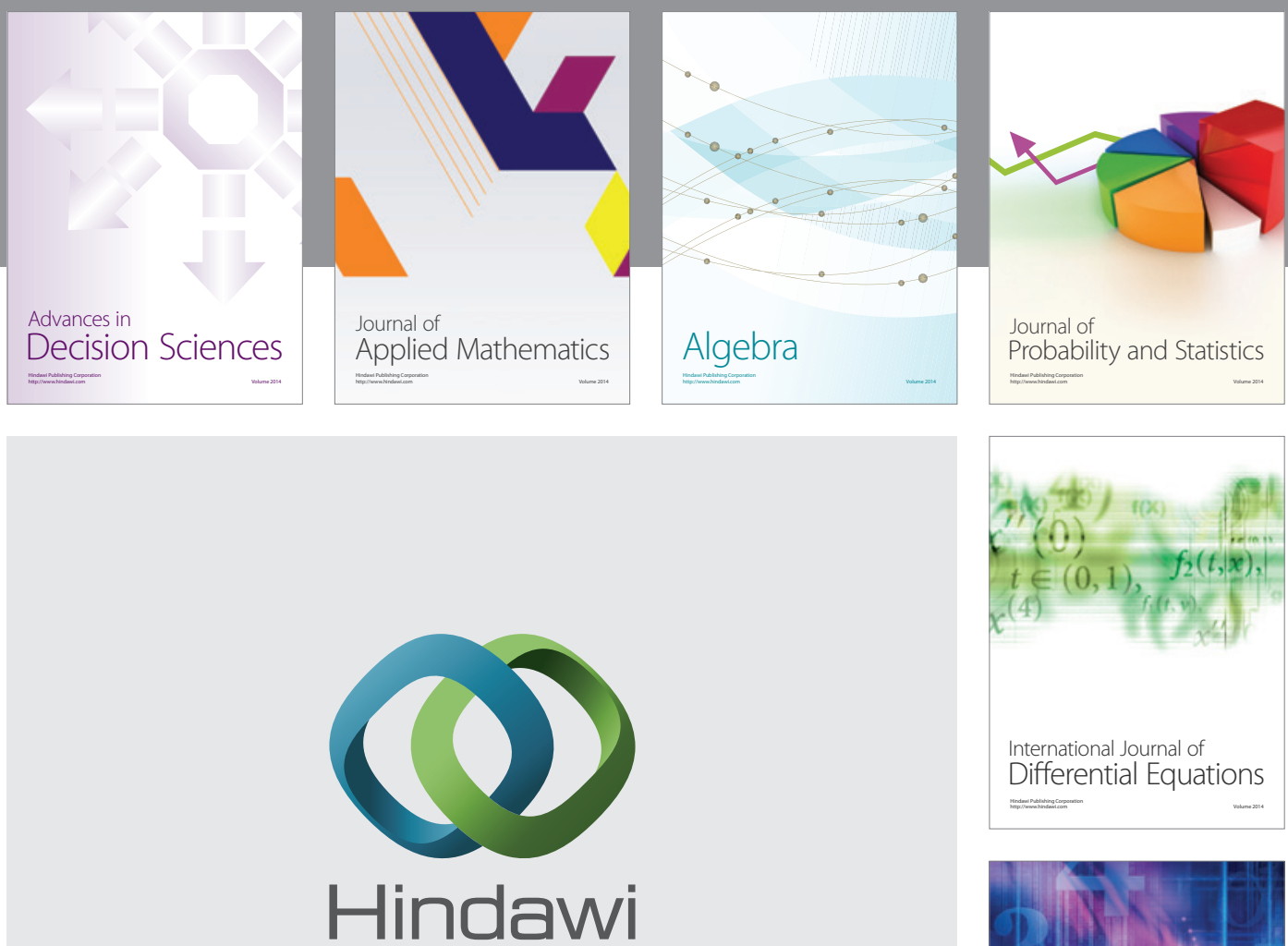

Submit your manuscripts at http://www.hindawi.com
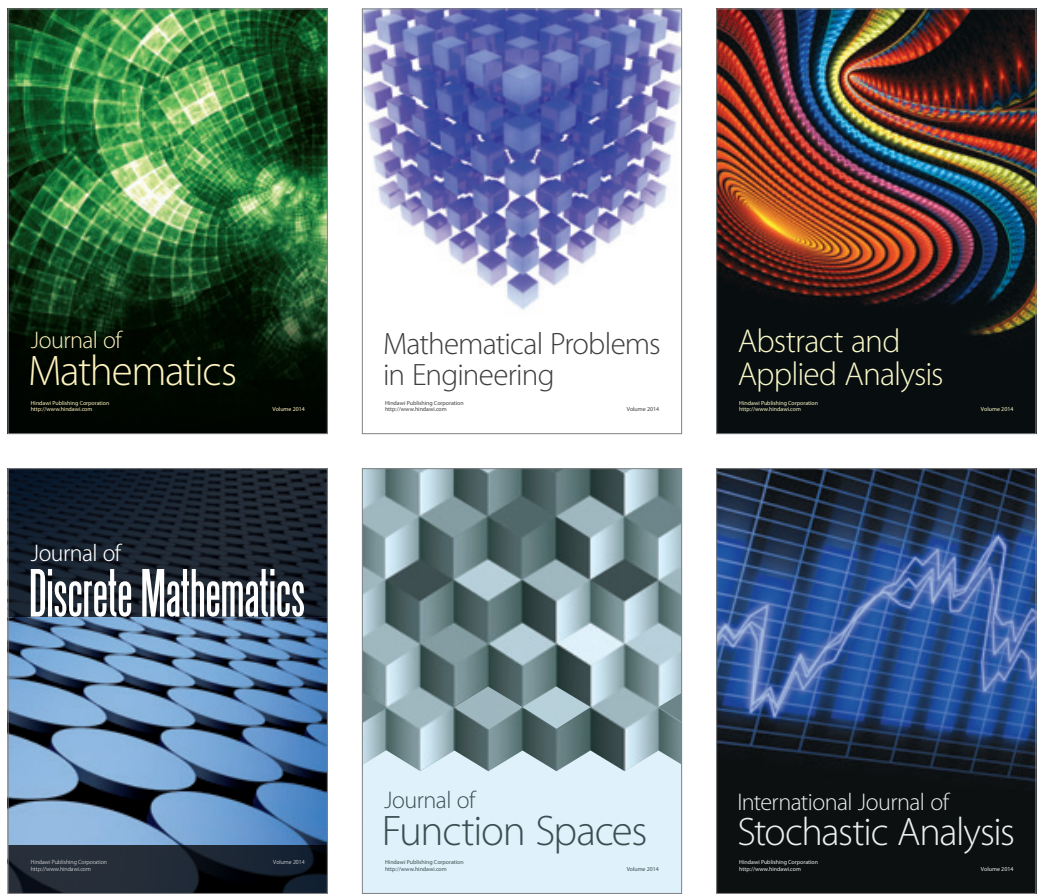

Journal of

Function Spaces

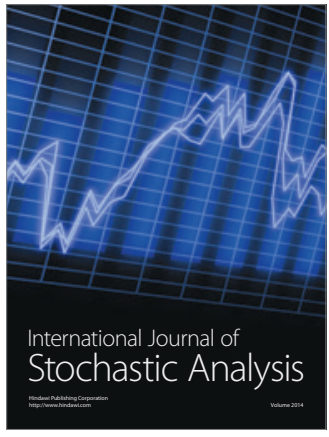

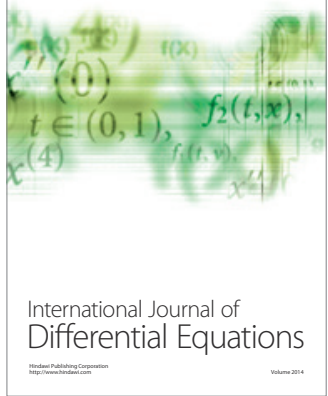
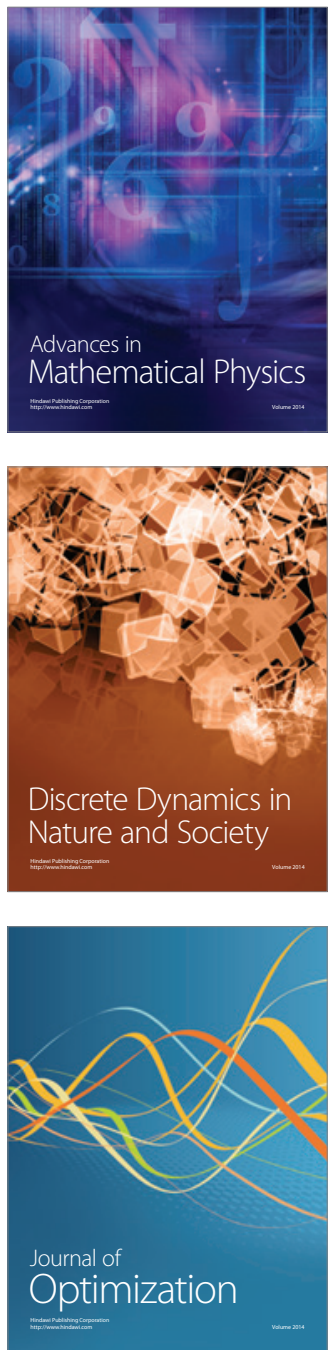